\title{
Characterization of the 3-HKT gene in important malaria vectors in India, viz., Anopheles culicifacies and Anopheles stephensi (Diptera: Culicidae)
}

\author{
R Vidhyanandhini, N Pradeep Kumar/ ${ }^{+}$ \\ Vector Control Research Centre, Indian Council of Medical Research, Medical Complex, Indira Nagar, 605006 Pondicherry, India
}

The 3-hydroxykynurenine transaminase (3-HKT) gene plays a vital role in the development of malaria parasites by participating in the synthesis of xanthurenic acid, which is involved in the exflagellation of microgametocytes in the midgut of malaria vector species. The 3-HKT enzyme is involved in the tryptophan metabolism of Anophelines. The gene had been studied in the important global malaria vector, Anopheles gambiae. In this report, we have conducted a preliminary investigation to characterize this gene in the two important vector species of malaria in India, Anopheles culicifacies and Anopheles stephensi. The analysis of the genetic structure of this gene in these species revealed high homology with the An. gambiae gene. However, four non-synonymous mutations in An. stephensi and seven in An. culicifacies sequences were noted in the exons 1 and 2 of the gene; the implication of these mutations on enzyme structure remains to be explored.

Key words: 3-hydroxykynurenine transaminase gene - xanthurenic acid - Anopheles

The role of different molecules in the development of Plasmodium parasites in Anopheline mosquitoes has been well studied (Siden-Kiamos \& Louis 2004). The most important among these is xanthurenic acid (XA), which plays a role in the exflagellation of male gametocytes in the midgut of Anopheles (Billker et al. 1998, Garcia et al. 1998). This molecule is the by-product of tryptophan metabolism. It acts as a chemical trigger, which is used by the malaria parasite for development inside the mosquitoes (Han et al. 2002). It plays a key role in parasite gametogenesis and fertility (Rossi et al. 2006).

Mosquitoes have evolved two alanine glyoxylate aminotransferases (AGT) (Han et al. 2006b). One is the cloned Aedes aegypti 3-hydroxykynurenine transaminase (AeHKT) (Han et al. 2002), which is homologous to human AGT (hAGT) and primarily catalyzes the transamination of 3-HK to XA in mosquitoes. The other is a typical dipteran AGT, which primarily catalyzes the conversion of glyoxylic acid to glycine (Han et al. 2006a). 3-HK is the metabolic intermediate of the tryptophan degradation pathway (Rossi et al. 2005, 2006).

The 3-HKT gene in the vector host plays a vital role in the development of Plasmodium (Han et al. 2002). This gene has already been implicated in the development of the Plasmodium species in the most important global malaria vector, Anopheles gambiae. In An. gambiae, the vector for the most deadly malarial parasite Plasmodium falciparum, XA plays a key role in the parasite gametogenesis and fertility (Rossi et al. 2006).

+ Corresponding author: dr_n pradeepkumar@hotmail.com Received 25 January 2008

Accepted 24 June 2008
The 3-HKT gene in the mosquito has been identified as a promising candidate gene towards discovery of novel malaria transmission-blocking drugs (Rossi et al. 2006). However, there is virtually no information on this gene in Anopheles stephensi and Anopheles culicifacies, important malaria vectors in India. Characterization of the 3-HKT gene in An. culicifacies and An. stephensi population is of utmost importance in devising alternative control strategies. Hence, a preliminary study with the goal of amplification of the 3-HKT gene was undertaken to study this gene in An. culicifacies and An. stephensi collected from South India, Pondicherry (Union Territory) and Rameswaram (Tamil Nadu).

DNA was extracted from field-collected and morphologically identified (Barraud 1934) specimens of $A n$.

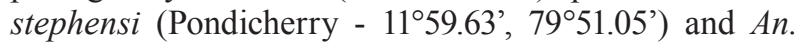
culicifacies (Rameswaram - 09¹5.24', 79¹6.44') using standard protocol (Collins et al. 1987). The pellet containing the DNA was air-dried for $30 \mathrm{~min}$ and dissolved in de-ionized Sigma grade water. A portion of the isolated DNA was stored in the DNA Bank maintained at VCRC.

The DNA primers designed based on An. gambiae genome sequences (GenBank acc. n. NT_078267) using "Primer 3" software (available at http://frodo-wi.mit.edu/cgi-bin/primer3/ primer3 www.cgi); viz., 5' -ATTCCGGAAAAGATAATGATCG-3' ${ }^{\prime}$ and 5' -TTCATCATAACAGCCCCAATAA-3', were used to amplify the 3 -HKT gene. The reaction mixture $(50 \mu \mathrm{L})$ consisted of the following components: $10 \mathrm{mM}$ dNTP (Finnzymes, Finland), $50 \mathrm{mM} \mathrm{MgCl}_{2}$ (Finnzymes, Finland), 10 pmol each of forward and reverse primers (Metabion, Germany), 10X buffer, 3 units Taq DNA polymerase (Finnzymes, Finland) and 10 ng template DNA made up with de-ionized water. The reaction was mixed in a $200 \mu \mathrm{L}$ PCR tube and PCR was performed on a Master Cycler Gradient (Eppendorff, Germany). The program consisted of an initial denaturation of $95^{\circ} \mathrm{C}$ for $5 \mathrm{~min}$ for An. stephensi and $4 \mathrm{~min}$ for An. culicifacies, 
followed by 36 cycles of denaturation at $94^{\circ} \mathrm{C}$ for $50 \mathrm{~s}$, annealing at $51^{\circ} \mathrm{C}$ for $A n$. stephensi and $53^{\circ} \mathrm{C}$ for $A n$. culicifacies for $1 \mathrm{~min}$ and extension at $72^{\circ} \mathrm{C}$ for $1 \mathrm{~min}$ $10 \mathrm{~s}$ with a final extension step of $72^{\circ} \mathrm{C}$ for $7 \mathrm{~min}$. The amplified products were detected by running them on a $1 \%$ agarose gel with $100 \mathrm{bp}$ molecular weight marker (Invitrogen, USA). The gels were stained with ethidium bromide, observed under a UV transiluminator, and then photographed using an Alpha Innotech Gel Documentation system.

The size of the amplified fragment of the 3-HKT region of An. culicifacies and An. stephensi was found to be approximately $800 \mathrm{bp}$ (Fig. 1). The PCR products were purified by QIA quick (Qiagen, Germany) PCR purification kit and sent for custom sequencing in both forward and reverse directions.

The DNA sequences of the 3-HKT gene were amplified and submitted to GenBank. This gene was $763 \mathrm{bp}$ for An. culicifacies (EF525557) and 766 bp for An. stephensi (EF520342). Both of the sequences were found to have high sequence homology with the An. gambiae 3 -HKT gene in the BLAST homology analysis. The maximum homology of the An. culicifacies 3-HKT gene sequence with the An. gambiae partial mRNA for 3-HKT (XM_311559) was $84 \%$, with $91 \%$ query coverage. An. stephensi 3-HKT and An. gambiae 3-HKT partial mRNAs (XM_311559) were $83 \%$ and $79 \%$, respectively. The GC contents of An. stephensi and An. culicifacies 3 -HKT were $58.04 \%$ and $48.75 \%$, respectively. Deduced amino acid sequences showed the presence of four non-synonymous mutations for An. stephensi and seven for An. culicifacies. This may result in amino acid changes in the protein structure. However, the ratio between the nucleotide diversities in non-synonymous and synonymous positions $\left(d_{\mathrm{NS}} / d_{\mathrm{S}}\right.$ ratio) was below unity in both species (0.0686 and 0.0357 for An. culicifacies and An. stephensi, respectively), clearly showing a negative selection pressure against protein change (Nei 1987).

Phylogenetic analysis (Neighborhood Joining method) was carried out using MEGA 4.0 software (Tamura et al. 2007) to understand the evolutionary pattern of the 3-HKT gene among different mosquitoes for which DNA sequences are available (Fig. 2). The phylogenetic tree indicated a clustering of An. stephensi and An. culicifacies with the An. gambiae 3-HKT gene (NT_078267). The pairwise Kimura 2 Parameter (Kimura 1980) genetic distance estimated with the available sequences of 3HKT gene is given in Table. It was found that the 3-HKT gene sequences of An. culicifacies were closely related to An. stephensi; the genetic distance between these being only 0.199 compared to An. gambiae (0.216). The maximum genetic distance recorded was 0.494 between An. culicifacies and Ae. aegypti.

The 3-HKT gene in An. gambiae consists of four exons and three introns and is $1439 \mathrm{bp}$ long. The structure of the gene is: exon 1 (669 bp), intron 1 (70 bp), exon 2 (95 bp), intron 2 (72 bp), exon 3 (249 bp), intron 3 (70 bp) and exon 4 (214 bp). The amplified portions of the gene sequences of An. stephensi and An. culicifacies in this study include only the first exon, the first intron and a portion of the sond exon. The analysis of the structure of the gene sequences of this enzyme in these species revealed high homology with the An. gambiae gene. However, six non-synonymous mutations were recorded in An. culicifacies (Q51R, D92E, T113E, A126P, T136A and R221K), while only four non-synonymous mutations were seen in An. stephensi (T113E, A126P, T136A and V168I), compared to An. gambiae. In addition, another non-synonymous mutation in the second exon K227R was also found in An. culicifacies compared to An. gambiae (Fig. 3). The implications of these non-synonymous mutations are currently being explored.

The size of the first intron was also found to be variable between these Anopheline species. While the size of the intron was only $68 \mathrm{bp}$ in An. culicifacies, it was longer (83 bp) in An. stephensi, compared to $70 \mathrm{bp}$ for An. gambiae.

Manipulating the expression of 3-HKT molecules in the mosquito species would block the development of sexual stages of the parasite inside the mosquito vector and may, therefore, represent a promising avenue for the discovery of novel malaria transmission-blocking drugs (Rossi et al. 2006). This study provides evidence for the 3-HKT gene in An. stephensi and An. culicifacies, important mosquito vectors of malaria in India. Further

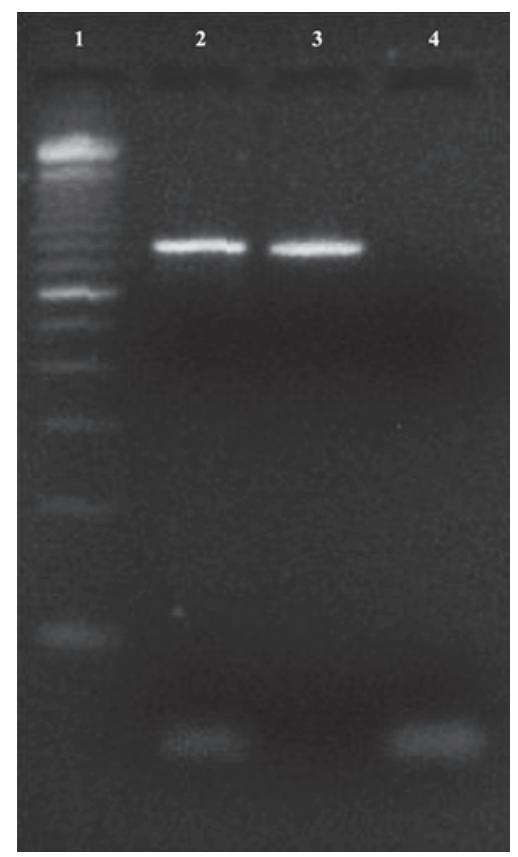

Fig. 1: amplification of 3-HKT gene from An. stephensi and An. culicifacies mosquito from Pondicherry and Rameswaram, India; Lane 1: mol. Weight marker; Lane 2: An. stephensi, Pondicherry; Lane 3: An. culicifacies, Rameswaram; Lane 4: negative control.

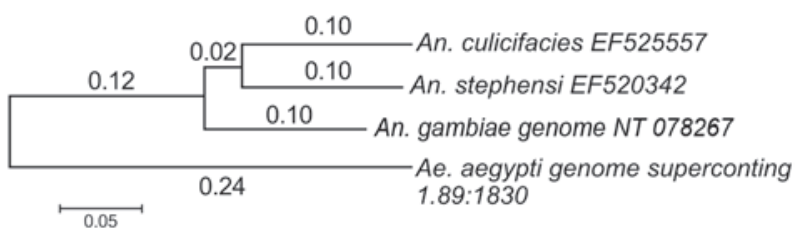

Fig. 2: phylogeny tree of 3-HKT sequences of Anopheles mosquitoes and Ae. aegypti. 
TABLE

Pairwise genetic distances (Kimura 2 parameter) of 3-HKT gene among different species of mosquitoes

\begin{tabular}{lcccc}
\hline Species & Ae. aegypti & An. gambiae & An. culicifacies & An. stephensi \\
\hline Ae. aegypti & - & & & \\
An. gambiae & 0.461 & - & - \\
An. culicifacies & 0.494 & 0.216 & 0.199 \\
An. stephensi & 0.475 & 0.226 & - \\
\hline
\end{tabular}

Exon 1:

An. gambiae NT_078267.3

An.stephensi Br520342

An.culicifacies EF525557

An. gambiae NT_078267.3

An.stephensi EF520342

An.culicifacies EF525557

An.gambiae NT_078267.3

An.stephensi B̈F520342

An.culicifacies EF525557

Intron 1:

An.gambiae NT_078267.3

An.stephensi Br520342

An.culicifacies EF525557

Exon 2:

An. gambiae NT 078267.3

An. stephensi B̈F520342

An.culicifacies EF525557

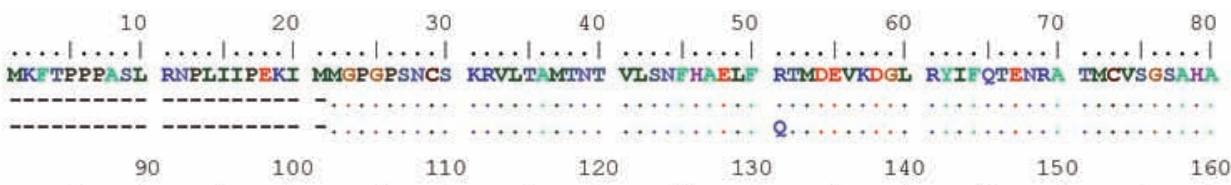

$\ldots|\ldots| \ldots|\ldots| \ldots|\ldots| \ldots|\ldots| \ldots|\ldots| \ldots|\ldots| \ldots|\ldots| \ldots|\ldots| \ldots|\ldots| \ldots|\ldots| \ldots \mid$

GMEAMLSNLL EEGDRVIIAV NGIWAERAVE MSERYGADVR TIEGPPDRPE SLETLARAIE IHQPKCLIIT HGDSSSGLLQ

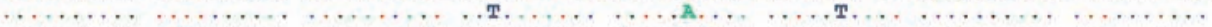

…

$\begin{array}{llllll}170 & 180 & 190 & 200 & 210 & 220\end{array}$

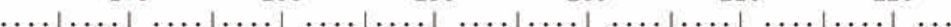

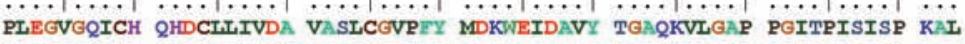

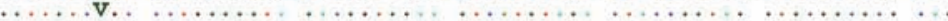

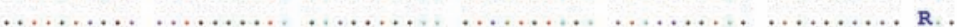

Fig. 3: deduced amino acid sequences of 3-HKT gene amplified for An. culicifacies and An. stephensi.

characterization of 3-HKT gene products would yield a better insight into the role of 3-HKT in the development of Plasmodium parasites in these vector mosquitoes.

\section{ACKNOWLEDGEMENTS}

To Dr. S. Sabesan, Deputy Director (Senior Grade) and Chief HRD, Dr. P. Jambulingam, Deputy Director (Senior Grade), for their consent and encouragement to undertake this study, and Shri. N. Krishnamoorthy and Shri. P. M. Ajithlal, $\mathrm{VCRC}$, for their technical assistance.

\section{REFERENCES}

Barraud PJ 1934. Fauna of British India including Ceylon and Burma, Diptera, Vol. V, Family Culicidae, Taylor and Francis, London, $513 \mathrm{pp}$.

Billker O, Lindo V, Panico M, Etiene AE, Paxton T, Dell A, Rogers M, Sinden RE, Morris HR 1998. Identification of xanthurenic acid as the putative inducer of malaria development in the mosquito. Nature 392: 227-228.

Collins FH, Mendez MA, Rasmussen MO, Mehaffey PC, Besansky NJ, Finnerty V 1987. A ribosomal RNA gene probe differentiates member species of the An. gambiae complex. Am J Trop Med Hyg 37: $37-41$.

Garcia GE, Wirtz RA, Barr JR, Woolfitt A, Rosenberg R 1998. Xanthurenic acid induces the gamatogenesis in Plasmodium, the malaria parasite. J Biol Chem 273: 12003-12005.

Han Q, Fang J, Li J 2002. 3-hydroxykynurenine transaminase iden- tity with alanine glyoxylate transaminase. $J$ Biol Chem 277: 15781-15787.

Han Q, Kim S R, Ding H, Li J 2006a. Evolution of two alanine glyoxylate aminotransferases in mosquito. Biochem J 397: 473-481.

Han Q, Robinson H, Gao YG, Vogelaar N, Wilson SR, Rizzi M, Li J 2006b. Crystal structures of Aedes aegypti alanine glyoxylate aminotransferase. J Biol Chem 281: 37175-37182.

Kimura M 1980. A simple method for estimating evolutionary rate of base substitutions through comparative studies of nucleotide sequences. J Mol Evol 16: 111-120.

Nei M 1987. Molecular evolutionary genetics. Columbia University Press, New York, 512 pp.

Rossi F, Li J, Rizzi M, Garavaglia S, Arca B, Giovenzana GB 2006. Crystal structure of the Anopheles gambiae 3-hydroxykynurenine transaminase. Proc Natl Acad Sci USA 103: 5711-5716.

Rossi F, Lombardo F, Paglino A, Cassani C, Miglio G, Arca B, Rizzi $M$ 2005. Identification and biochemical characterization of the Anopheles gambiae 3-hydroxykynurenine transaminase. FEBS J 272: 5653-5662.

Siden-Kiamos I, Louis C 2004. Interaction between malaria parasites and their mosquito hosts in the midgut. Insect Biochem Mol Biol 34: 679-685.

Tamura K, Dudley J, Nei M, Kumar S 2007. MEGA4: Molecular Evolutionary Genetics Analysis (MEGA) software version 4.0. Mol Biol Evol 24: 1596-1599. 